\title{
Do imagined and executed actions share the same neural substrate?
}

\author{
Jean Decety ${ }^{*}$ \\ INSERM Unit 94, 16 Avenue du Doyen Lépine, F-69500 Bron, France
}

Accepted 22 August 1995

\begin{abstract}
This paper addresses the issue of the functional correlates of motor imagery, using mental chronometry, monitoring the autonomic responses and measuring cerebral blood flow in humans. The timing of mentally simulated actions closely mimic actual movement times. Autonomic responses during motor imagery parallel the autonomic responses to actual exercise. Cerebral blood flow increases are observed in the motor cortices involved in the programming of actual movement (i.e. premotor cortex, anterior cingulate, inferior parietal lobule and cerebellum). These three sources of data provide converging support for the hypothesis that imagined and executed actions share, to some extent, the same central structures.
\end{abstract}

Keywords: Motor imagery; Cognitive motor process; Autonomic response; Mental chronometry; Brain activation

\section{Introduction}

In the framework of the cognitive neuroscience approach, which combines concepts and methods of cognitive psychology and of neuroscience, accumulated data provide strong support for the central assumption of the current conception of motor control. The organization of motor activity is supposed to be based upon the utilization by the motor system of information stored in memory in the form of multiple hierarchically organized representations of action [44]. Thus, there is common agreement that motor acts are centrally represented, and, like other representations, are stored, modified, and may be retrieved through specific cognitive processing [49]. Furthermore, it is generally thought that cognitive processes and overt behaviour are intimately related.

Motor imagery can be defined as a dynamic state during which a subject mentally simulates a given action. This type of phenomenal experience implies that the subject feels himself performing a given action. It corresponds to the so called internal imagery (or first person perspective) of sport psychologists [33]. Converging evidence from several sources indicates that motor imagery pertains to the same category of processes as those which are involved in programming and preparing actual actions, with the difference that in the latter case execution would be blocked at

\footnotetext{
* Corresponding author. Fax: (33) (72) 369760.
}

some level of the cortico-spinal flow [6]. There is now repeated evidence that motor imagery has significant positive effects on motor skill learning $[14,19]$.

The same reasoning that is used in visual imagery research $[20,29]$ can be extended to motor imagery, by assuming that motor images share the same neural mechanisms as those that are also responsible for preparation and programming of actual movements [6].

The central hypothesis leading to this research is the following: do motor images share the same neural mechanisms as those that are also responsible for preparation and programming of actual movements? Three methods have been used to tackle this question: mental chronometry, monitoring autonomic responses and measuring cerebral blood flow in normal subjects during motor imagery tasks.

\section{Experimental}

\subsection{Mental chronometry}

The use of the term 'mental chronometry' has no vitalistic implications, we believe that mental activities are represented by neural activities, although the presence of the former must be inferred from observational behaviour. The paradigm used in motor imagery is derived from the method introduced by Donders [18]. Donders measured the time employed by subjects to respond to sensory stimuli. He found that when the task required a stimulus discrimi- 
nation or a reaction choice, the reaction time was longer than when only one stimulus was presented and a predetermined response was required. Since that time, mental chronometry measures have been taken to reflect the level of efficiency of neural and cognitive mechanisms [34]. In our context, this method is a way of accessing the content of motor images.

Already in 1962, Landauer found similar completion times for implicit and explicit speech [31]. This leads him to suggest that the two behaviors might be involved in the same central processes. The comparison between actual and mental movement time has also been tested by Parsons [37] with a paradigm in which subjects moved one of their hands into the orientation of a stimulus (a hand) or mentally simulated that action. The results showed that mental simulation time mimicked movement time for natural efficient movement.

In a first experiment, we [3] compared the temporal organization of graphic movements executed either actually or mentally in normal subjects. The subjects had to perform two graphic tasks, drawing a cube or writing a sentence, with either the right (dominant) or the left hand. These tasks were selected because both of them are essentially accomplished by distal portions of the limbs and can therefore be attributed mostly to contralateral hemisphere motor control. Furthermore, these tasks allow a comparison between right and left hemisphere cognitive processing since handwriting involves left hemisphere resources while drawing is supposed to require more the participation of the right hemisphere visuo-spatial ability. This assumption is based on the fact that the functional specialization of the cerebral hemispheres is a prominent contributor to hand differences in performance [52]. The results showed that movement times were very stable from trial to trial in the same subject in both modalities (actual and mental). Furthermore, the time required to execute actual graphic gesture and the time to execute the same gesture mentally were similar. The mental left hand was found to be slower than the mental right hand in the same proportion to actual left hand and actual right hand. However, while this was significant for all subjects for the writing task, it was just a tendency for the drawing task. Yet, drawing a cube is a particular task which requires less graphic skill than does writing and is also supposed to be controlled by the right hemisphere. This suggest that the hands, in both mental and actual modalities, are stressed by two different factors, the hemispheric specialization of the task and the manual proficiency.

The same paradigm was applied by Decety and Boisson [5] to a group of neurological patients who were selected on the following criteria: unilateral lesions on computerised tomography, absence of aphasic, apraxic and verbal comprehension impairments. None of them showed an impairment in visual orientation or in visual imagery. The group consisted of three patients with right-sided hemiplegia, three patients with left-sided hemiplegia. Two patients with paraplegia and two patients with tetraplegia were also studied as a control group. In hemiplegic patients a significant difference in mental duration times was found between the paralyzed and the healthy 'represented limb'. The paralyzed limb was mentally much slower than the normal one. In contrast, mental movement times in tetraplegic and paraplegic patients did not differ from those in normal volunteers. Hence, high level motor processes, presumably cortically localized, appear to interact with the information processing units which underlie the mental representation of motor behaviour.

A second experiment [4] implied again a direct comparison of the duration of movements actually performed with that of movements mentally represented by the same subjects. A simple walking task was used, where blindfolded subjects were requested, either to walk, or to imagine themselves walking, to previously inspected targets. Walking times increased with target distance, in both the actual and the mental walking conditions, a finding which replicates and expands that of Kosslyn et al. [28] and Denis and Cocude [15] in their visual scanning experiments. In addition, and most importantly, walking times were invariant across actual and mental conditions. It might be, as has been suggested by several authors, that our subjects had tacit knowledge of what should happen when they had to walk longer distances, namely, that duration of the action should increase. If this were the case, the observed temporal invariance could be simply due to a strategy of the subjects of replicating in the mental condition the temporal sequence registered in the actual condition $[43,45,35]$. One possible way to answer this question is to introduce an external constraint on the motor task, such that the subjects would have to produce a greater effort to execute the same task. It was conjectured that, if time was the variable represented in the motor image, this external constraint should not affect the duration of the imagined action. If, on the other hand, other variables, like muscular force, were represented, then the durations of the imagined and the actually executed movements should differ, because the constraint would exert its effect only in the actual condition and not in the mental condition.

A second experimental session was thus performed in the same subjects, by using a condition involving an external constraint on movement execution. The constraint was a heavy load carried by the subjects while they actually walked or imagined themselves walking to the targets. The effects of this constraint were observed on motor timing. Walking times in the actual condition with the load were in the same range as those measured in the same subjects in the first experiment. By contrast, travel times in the mental walking condition with the load were significantly increased in all subjects and for all target distances. The results from the second session showed a clear dissociation between actual and mental walking times. It took the subjects carrying the load about $30 \%$ longer to mentally walk to the targets than to actually perform the 
task. Because actual walking times with the load remained virtually the same as without the load, the difference between actual and mental times was entirely due to an increase in the mental time. The fact that the similarity of actual and mental walking times obtained in the first session could be broken down in the second one demonstrates that subjects were not merely replicating in the mental walking task the duration they had experienced in the actual walking task [4].

In a third experiment, subjects were instructed either to actually walk or to imagine themselves walking on beams that had the same length but varied in width. The beam width was assumed to be a factor of difficulty, i.e. the narrower the beams, the more difficult the task. A significant effect of task difficulty was observed in both actual and mental movement times [8]. This result can be interpreted within the framework of the speed-accuracy trade-off which accounts for the duration of movements under accuracy constraints [21].

A recent experiment was designed to reinvestigate the validity of Fitts law in mentally simulated actions. Naive subjects were instructed to imagine walking in a three-dimensional virtual environment through gates of different apparent widths positioned at different apparent distances. Both the distance and the width of the virtual gates affected the mental response time. The time for mentally walking increased for increasing gate distances and for decreasing gate widths. Fig. 1 illustrates this result on group data.

In the present context, the work of Georgopoulos and Massey [23] is of special interest again. In their study,

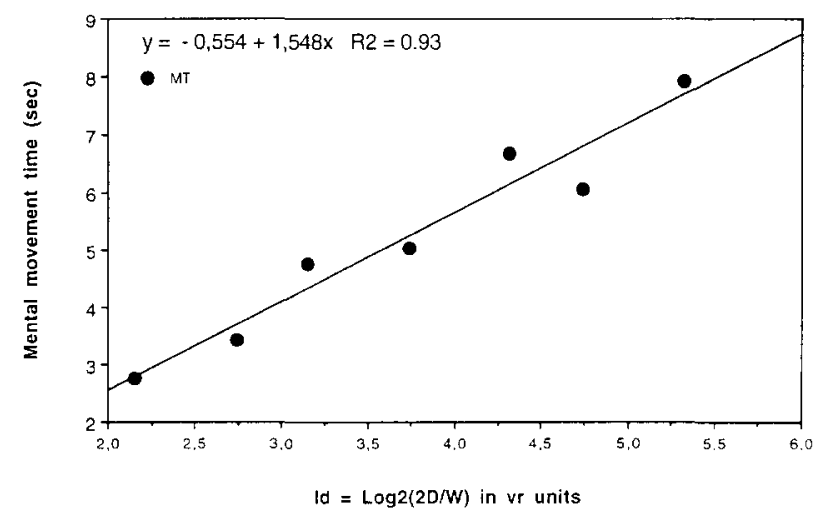

Fig. 1. Index of task difficulty (Id) plotted against mental movement time in 15 subjects required to imagine themselves walking in a virtual environment through gates of different apparent widths placed at different apparent distances. Raw data were computed using the Fitts'law formalisation which relates movement time $(M T)$ to the index of difficulty (Id) of the task, where $D$ is the gate distance, and $W$ the target width. Fitts' law is expressed as the relation: $M T=a+b \log _{2} 2 D / W . I d=\log _{2}$ $2 D / W$, where $a$ and $b$ are two constants. Assuming that this law was valid in our experimental conditions, virtual reality units (from the VR computer) were attributed to the apparent distances of the gates ( $D=100$, $200,300)$ and to their apparent widths ( $W=15,30,45)$. Empirical values of MT were found to correlate with the estimated $I d$, as follows: $M T=-0.554+1.548 I d\left(r^{2}=0.94\right)$. normal subjects were asked to move a manipulandum on a plane in directions other than going straight towards a visual stimulus. They were instructed to generate a movement at an angle from a stimulus direction which varied in 2-dimensional space from trial to trial. The direction of the movement and the reaction time were measured. The results showed that, in accordance with the Fitts' law, the increase in reaction time, which can be considered to be a mental movement time, was a linear function of the task difficulty (calculated from the angle achieved and its variability. This indicates that Fitts' law holds for the hypothesized rotatory motion of the imagined movement vector, and that both real and imagined movements might be governed by similar amplitude-accuracy relations.

Chronometric studies suggest that executive movement structures would be subordinated in the hierarchy to mental structures where the concepts underlying any preplanned action sequence would be represented. Thus, the content of these mental structures should logically be the same whether the executive structures are activated or not.

\subsection{Autonomic responses}

If motor imagery shares neural mechanisms that are also responsible for motor programming, as suggested by mental chronometry, then brain activation during imagined action should be reflected in some way at the level of peripheral effectors. Because autonomic activation may occur in many situations involving cognitive or affective arousal, it can be conjectured that, in order to be considered as a specific effect, the degree of vegetative activation during motor imagery should be proportional to the imagined effort.

In order to test the foregoing hypothesis of a commonality of neural structures involved in imagining and in programming actions, an experiment was conducted that systematically varied the amount of mental effort produced by the subject [9]. Measurements of cardiac and respiratory activity during actual and mental locomotion at increasing speeds revealed a covariation of heart rate and pulmonary ventilation with the degree of imagined effort. The degree of vegetative activation of a subject mentally running at 12 $\mathrm{km} / \mathrm{h}$ was comparable to that of a subject actually walking at $5 \mathrm{~km} / \mathrm{h}$. The autonomic response to imagined exercise was since confirmed by Wang and Morgan [55]. In their study, the subjects were instructed to imagine lifting dumbbells. Their results indicated that imagery produced a significant increase in ventilation, as well as significant elevations in systolic blood pressure. However, one cannot rule out a possible contribution of peripheral metabolic changes to the observed autonomic effects during motor imagery. Indeed, it could have been that, during motor imagery tasks, the subjects had produced a proportional increase in muscular activity by co-contracting antagonist muscles groups. In order to dismiss a contribution of peripheral mechanism, a new experiment was designed, in 
which intramuscular metabolites $\left(\mathrm{Pcr}, \mathrm{P}_{\mathrm{i}}\right)$ and $\mathrm{pH}$ were non-invasively measured directly by nuclear magnetic resonance (NMR) spectroscopy and cardiorespiratory changes monitored in healthy subjects during both actual and mental leg exercise at two levels of work [11]. During actual exercise, heart and respiration rates increased, first abruptly and then gradually in relation to the level of work. End-tidal $\mathrm{pCO}_{2}$ was unaltered. NMR spectra showed a drop in phosphocreatine $(\mathrm{PCr})$ and an increase in inorganic phosphate $\left(\mathrm{P}_{\mathrm{i}}\right)$ concentrations. Intracellular $\mathrm{pH}$ fell to 6.65 at maximal effort with a $19 \mathrm{~kg}$ load. During motor imagery, both heart and ventilatory rate increased immediately after mental exercise was begun. This increase was proportional to the amount of simulated exercise. Heart rate remained about $25 \%$ below the level observed during actual exercise. The increase in respiration rate, by contrast, was more marked than during actual exercise. Finally, end-tidal $\mathrm{pCO}_{2}$ decreased progressively to about $18 \%$ of the resting value. No changes in NMR spectra were detected with respect to the resting values. Although this method does not indicate to what extent a limited increase in EMG activity could be present during mental simulation, such activity is unlikely to explain the observed changes in ventilation. This study was replicated by Wuyam et al. [56] in subjects required to imagine walking on a treadmill at different speeds, and showed a significant increase in ventilation and a concomitant fall in end-tidal $\mathrm{pCO}_{2}$.

Monitoring of autonomic changes thus demonstrates that the vegetative activation during motor imagery is greater than required by the increase in metabolic demands and, therefore, that a significant fraction of this vegetative activation has to be of a central origin. The fact that vegetative activity during mental effort increases beyond the level of metabolic demands supports our initial hypothesis of a commonality of mechanisms between actual and imagined action. This effect can be considered as a vegetative response pertaining to the normal pattern of activation which occurs during motor programming. In this way the central programming structures would anticipate the need for energetic mobilization required by the planned movement to the same extent as they anticipate the amount of activity needed in the motor pathways for producing the movement.

Another argument favoring the hypothesis that cardiorespiratory changes during mental simulation of movements are centrally controlled can be drawn from investigating cardio-respiratory changes during motor imagery in heart-lung transplants. Indeed, recipients of heart-lung transplants represent an unusual opportunity to evaluate the importance of central activation of autonomic functions during mental simulation of motor activity. These patients have denervated afferents and efferents to their hearts and loss of pulmonary afferents. As predicted, heart-lung transplants exhibited an appropriate ventilatory response, but no cardiovascular response because of the effects of cardiac denervation as compared to healthy volunteers [13].

\subsection{Measurements of regional cerebral blood flow}

The initial hypothesis that motor images share, to some extent, the same neural mechanisms as those that are also responsible for preparation and programming of actual movements is thus supported by mental chronometry as well as by autonomic responses. Other confirmatory arguments can be drawn from experiments using functional mapping of the regional cerebral blood flow ( $\mathrm{rCBF}$ ) which is taken as an index of neuronal activity.

Ingvar and Philipsson [26] measured rCBF for the first time in human subjects who were instructed either to imagine a clenching hand movement with a slow rhythm, or actually to carry out the same movement. During mental simulation they found significant flow increases in the premotor and frontal regions. When the hand movements were actually carried out there was mainly an activation of the same magnitude in the contralateral primary motor cortex. Roland and collaborators [46] asked normal subjects to imagine a rapid and skilled sequence of digit movements. They found a significant and localised rCBF change mainly in the supplementary motor area (SMA). Their results thus confirmed that the SMA plays an important role for the internal programming and simulation of complex motor sequences. Measuring rCBF with ${ }^{133}$ Xenon and a gamma camera, Decety, Philippon and Ingvar [2] studied normal subjects during the execution or during the imagination of a graphic movement (writing 'one, two, three, etc.') either with the right or the left hand. During the imagery condition, the subjects were instructed to imagine the movement at the 'first person perspective' and to try to 'feel their writing hand'. During motor imagery, significant bilateral activations were detected in the prefrontal cortex, in the supplementary motor area, and also in the cerebellum. During the execution, the same regions were found to be activated with the addition of the primary motor and somatosensory cortices in the contralateral hemisphere. The participation of the cerebellum has been since confirmed. Using the single photon emission computed tomography (SPECT) during motor imagery of a tennis skill training in normal volunteers, Decety, Sjöholm, Ryding, Stenberg and Ingvar [7] observed a significant rCBF increase bilaterally in the cerebellum, as well as in the basal ganglia and in the premotor cortex. These findings were replicated by Ryding, Decety, Sjöholm, Stenberg and Ingvar [48] using the hexamethyl-propyleneamine oxyme (HMPAO) labelled with $99 \mathrm{mTc}$ as a blood flow tracer. Confirmation by other PET studies was provided by Stephan et al. [50] showing that imagined finger movements activate the premotor cortex, the SMA, the anterior cingulate as well as parietal areas bilaterally. Using the functional magnetic resonance technique, Tyszka et al. [53] reported activation of the SMA and of the anterior cingulate during imagination of finger movements.

In a recent experiment, $\mathrm{rCBF}$ was measured with PET in normal subjects during the mental simulation of grasp- 
ing movements [12]. The control condition from which the activation condition was subtracted consisted of the visual inspection of virtual objects (of different size, color and orientation) presented in subjects' reaching space. Significant activations were observed in areas concerned with motor behavior. At the cortical level, Brodmann area 6 in the inferior part of the frontal gyrus was activated on both sides with a stronger activation in the left hemisphere (contralateral to the hand mentally involved in the task). On the left, contralateral to the side of the simulated movement, activation was also observed in prefrontal areas, extending to the dorsolateral frontal cortex (areas 9, 8 and 46), and in the inferior parietal lobule (area 40). Finally, the anterior cingulate cortex (areas 24 and 32) was bilaterally activated. At the subcortical level, the caudate nucleus was found to be activated on both sides and the cerebellum was involved on the left side.

In this later study, the ventral and mesial portions of SMA were not found to be activated. Instead, more lateral premotor areas were involved. This discrepancy with previous studies can be explained by a difference in mental motor tasks: our task consisted in simulating visuallyguided movements, whereas SMA exhibits preferential activity during internally-guided tasks [36,51]. Finally, the activity in anterior cingulate cortex has been previously shown to be related to attention requiring tasks $[41,42]$. By subtracting the two conditions from each other, we completely suppressed activation of area 24 , whereas area 32 remained active in motor imagery. This finding confirms that part of anterior cingulate cortex plays an important role in higher motor control [39].

There are other brain imaging studies that support this hypothesis. Lang et al. [32] measured rCBF with PET during the execution of saccadic eye movements and during the mental simulation of the same task. During the mental condition, they found significant activations bilaterally in the SMA and precentral gyrus, and in the cingulate gyrus.

The above mentioned cortical as well as subcortical areas found to be activated during motor imagery pertains to the neural network known to be involved in the early stage of motor control (i.e. motor programming). This gives strong support to common neural mechanisms of imagery and motor preparation.

\section{Discussion}

The three sources of information presented in this paper (i.e. chronometric studies, $\mathrm{rCBF}$ and autonomic measurements) converge to support the notion that motor imagery shares the same neural mechanisms that are involved in motor control of actual actions.

Cognitive neuroscientists often describe three levels of computation in motor control [30]. At the coarest level, a path must be planned. At the next level the inverse kine- matics problem must be solved at each point along the path; one must compute the proper joint angles. And at the finest level, the inverse dynamics problem must be solved at each point; one must compute the muscle forces needed to move the joints into the proper positions. These levels are not necessarily independent. A decision at any one of these three levels has direct implications for decisions at the others.

On the basis of these points, one would like to suggest that motor imagery is controlled by those parts of the frontal cortex which are specifically involved in carrying out the computations of the action programming subsystem which deals with planning at the coarest level (responsible for the timing of cognitive and motor events). The evidence available shows that the prefrontal and the dorsolateral frontal cortex play a fundamental role in this timing $[41,47]$. Prefrontal cortex lesions can produce defects in " executive functions' in the control of behaviour - in planning, coordinating, or controlling a sequence of action [17]. It has been argued that the prefrontal cortex is necessary for regulating behaviour guided by representations or internalized models of reality by Goldman-Rakic [25]. Moreover, it is known from lesion studies in monkeys that the premotor cortex plays a crucial role in the retrieval of movement [38]. Therefore it seems relevant to find rCBF increases in that region during motor imagery. The cerebellar activation during motor imagery is congruent with the fact that there are anatomical connections between the prefrontal cortex (including the SMA) and the cerebellum, the basal ganglia and the ventral thalamic nuclei $[1,27]$. One could suggest that the activation of the cerebellum during motor imagery reflects an inhibitory mechanism which prevents the efferent impulses, triggered through imagery, to reach the medullar and muscle levels. As suggested by Pickenhain [40], mental simulation of motor behavior might trigger the motor program and involve all parts of the central nervous system which serve as subprogram effectors for the whole process of realization of a given action. Apparently this also includes autonomic mechanisms. However, it should be recalled that any outflow activity from the central nervous system also involves inhibitory processes. The formulation of a given motor plan, as well as the execution of it, requires rejection of irrelevant plans and concepts. Inhibition thus forms an important part of the general role played by the prefrontal cortex [22-25] in its simultaneous handling (selection/rejection) of temporally separate but mutually convergent serial action programs. Another argument supporting the hypothesis that motor imagery shares the same neural mechanisms as those that are responsible for preparation and programming of actual movements is given by the study of Yue and Cole [57]. In their experiment, the authors examined whether strength increases may result from training-induced changes in voluntary motor programs. They compared, in normal subjects, the maximal voluntary force production of the fifth digit's meta- 
carpophalangeal joint after a training program of repetitive maximal isometric muscle contractions and after a motor imagery training that did not involve repetitive activation of muscle. The average abduction force of the left (trained) digit increased $22 \%$ for the imagining group and $30 \%$ for the contraction group. The maximal abduction force of the right (untrained) fifth digit increased significantly in both the imagining and contraction groups after training. Thus, strength increases can be achieved without repeated muscle activation. These force gains appear to result from practice effects on central motor programming. Recently, Di Pellegrino, Fadiga, Fogassi, Gallese and Rizzolatti [16] have reported that premotor neurons can retrieve movements not only on the basis of stimulus characteristics but also on the basis of the meaning of the observed actions. Indeed, neurons of the rostral part of the inferior premotor cortex of monkeys were found to discharge during goal directed movements such as grasping, tearing and holding as well as when the monkeys observe the same specific meaningful hand movements performed by the experimenters. These neurons could well be labeled as 'representational neurons'.

These results altogether suggest that mental representations during observation of actions performed by others, and even more during simulation of one's own actions, share common neural mechanisms with other covert aspects of motor performance, such as planning and programming.

I should like to conclude this paper by suggesting three prospective lines for future research on the functional equivalence between the neural systems for actual and imagined movement.

The first one aims to fractionate the phenomenological experience of motor imagery into different component processes. Indeed, as Vogt [54] suggested, motor imagery needs preparation too. And motor preparation involves, to some extend, the same neural substrate $[10,50]$. The use of high resolution functional imaging technics (PET or fMRI) can split these regions into those involved with motor preparation and with internal simulation processes, respectively. The same reasoning is true for the phenomenological distinction between internal versus external imagery which is supposed to rely on visual and kinesthetic information processing.

The second deals with the relevant distinction between implicit and explicit cognitive processing which also applies to imagery. Mental simulation is clearly a process under voluntary control and requires consciousness. But motor imagery may also be involved in an implicit (nonconscious) way, such as the left-right handedness judgment paradigm used by Parsons [37]. It would be of interest to know the exact correspondence between the two processings.

Finally, experimental paradigm should try to separate the attentional component of motor imagery. Working memory and attention are mediated by the prefrontal cor- tex (area 46), anterior cingulate and thalamus. Part of the network that subserves motor imagery is similar [10]. Although motor imagery is made of working memory and attention it should be possible to dissociate these two processes.

\section{References}

[1] Allen, G.I. and Tsukahara, N., Cerebro-cerebellar communication systems, Physiol. Rez., 54 (1974) 957-1006.

[2] Decety, J., Philippon, B. and Ingvar, D.H., rCBF landscapes during motor performance and motor ideation of a graphic gesture, Eur. Arch. Psychiat. Neurol. Sci., 238 (1988) 33-38.

[3] Decety, J. and Michel, F., Comparative analysis of actual and menta movement times in two graphic tasks, Brain Cogn., 11 (1989) $87-97$.

[4] Decety, J., Jeannerod, M. and Prablanc, C., The timing of mentally represented actions, Behau. Brain Res., 34 (1989) 35-42.

[5] Decety, J. and Boisson D., Effect of brain and spinal cord injuries on motor imagery, Eur. Arch. Psychiat. Clin. Neurosci., 240 (1990) $39-43$.

[6] Decety, J. and Ingvar, D.H., Brain structures participating in mental simulation of motor behavior: a neuropsychological interpretation, Acta Psychol., 73 (1990) 13-34.

[7] Decety, J., Sjöholm, H., Ryding, E., Stenberg, G. and Ingvar, D. The cerebellum participates in mental activity: tomographic measurements of regional cerebral blood flow, Brain Res., 535 (1990) 313-317.

[8] Decety, J., Motor information may be important for updating the cognitive processes involved in mental imagery of movement, Eur. Bull. Cogn. Psychol., 4 (1991), 415-426.

[9] Decety, J., Jeannerod, M., Germain, M. and Pastène, J., Vegetative response during imagined movement is proportional to mental effort, Behav. Brain Res., 42 (1991) 1-5.

[10] Decety, J., Kawashima, R., Gulyas, B. and Roland, P.E., Preparation for reaching: a PET study of the participating structures in the human brain, NeuroReport, 3 (1992) 761-764.

[11] Decety, J., Jeannerod, M., Durozard, D. and Baverel, G., Central activation of autonomic effectors during mental simulation of motor actions in man, J. Physiol., 461 (1993) 549-563.

[12] Decety, J., Perani, D., Jeannerod, M., Bettinardi, V., Tadary, B., Woods, R., Mazziotta, J.C. and Fazio, F., Mapping motor representations with PET, Nature, 371 (1994) 600-602.

[13] Decety, J., Grezes, J., Wiesendanger, T., Faillenot, I., Rezig, M. and Benchetrit, G., Ventilatory and cardiac responses to actual exercise and to mental simulation in heart-lung transplants, submitted.

[14] Denis, M., Visual imagery and the use of mental practice in the development of motor skills, Can. J. Appl. Sport Sci., 10 (1985) 4-16.

[15] Denis, M. and Cocude, M., Scanning visual images generated from verbal descriptions, Eur. J. Cogn. Psychol., 1 (1989) 293-307.

[16] Di Pellegrino, G., Fadiga, L., Fogassi, L., Gallese, V. and Rizzolatti, G., Understanding motor events: a neurophysiological study, Exp. Brain Res., 91 (1992) 176-180.

[17] Ducan, J., Disorganisation of behaviour after frontal lobe damage, Cogn. Psychol., 3 (1986) 271-290.

[18] Donders, F.C., On the speed of mental processes (originally published in Dutch, translated by W.G. Koster), Acta Psychol., 30 (1969) 412-431.

[19] Feltz, D.L. and Landers, D.M., The effects of mental practice on motor skill learning and performance: a meta analysis, J. Sport Psychol., 5 (1983) 25-57. 
[20] Finke, R.A., Levels of equivalence in imagery and perception, Psychol. Rev., 87 (1980) 113-132.

[21] Fitts, P.M., The information capacity of the human motor system in controlling the amplitude of movement, J. Exp. Psychol., 47 (1954) 381-391.

[22] Fuster, J., The prefrontal cortex: anatomy, physiology, and neuropsychology of the frontal lobe, Raven Press, New York, 1980.

[23] Georgopoulos, A.P. and Massey, J.T., Cognitive spatial-motor processes, Exp. Brain Res., 65 (1987) 361-370.

[24] Goldberg, E. and Bilder, R.M., The frontal lobes and hierachical organization of cognitive control. In E. Perecman (Ed.), The Frontal Lobes Revisited, IRBN Press, New York, 1987.

[25] Goldman-Rakic, P.S., Circuitry of primate prefrontal cortex and regulation of behavior by representational memory. In V.B. Brooks and M.D. Bethesda (Eds.), Handbook of Physiology, the Nervous System V, 1987.

[26] Ingvar, D.H. and Philipson, L., Distribution of cerebral blood flow in the dominant hemisphere during motor ideation and motor performance, Ann. Neurol., 2 (1977) 230-237.

[27] Ito, M., The cerebellum and neural control, Raven Press, New York, 1984.

[28] Kosslyn, S.M., Ball, T.M. and Reiser, B.J., Visual images preserve metric spatial information: evidence from studies of image scanning, J. Exp. Psychol. Human Perc. Perf., 4 (1978) 47-60.

[29] Kosslyn, S.M., Seeing and imagining in the cerebral hemisphere: a computational approach, Psychol. Rev., 94 (1987) 148-175.

[30] Kosslyn, S.M. and Koenig, O., Wet Mind, The Free Press, New York, 1992.

[31] Landauer, T.K., Rate of implicit speech, Percept. Motor Skills, 15 (1962) 646.

[32] Lang, W., Petit, L., Höllinger, P., Pietrzyk, U., Tzourio, N., Mazoyer, B. and Berthoz, A., A positron emission tomography study of oculomotor imagery, NeuroReport, 5 (1994) 921-924.

[33] Mahoney, M.J. and Avener, M., Psychology of the elite athlete, Cogn. Ther. Res., 1 (1987) 135-141.

[34] Milner, A.D., Chronometric analysis in neuropsychology, Neuropsychologia, 24 (1986) 115-128.

[35] Mitchell, D.B. and Ritchman, C.L., Confirmed reservation: mental travel, J. Exp. Psychol. Human Perc. Perf., 6 (1980) 58-66.

[36] Mushiake, H., Inase, M. and Tanji, J., Neuronal activity in primate premotor, supplementary and precentral motor cortex during visually guided and internally determined sequential movements, J. Neurophysiol., 66 (1991) 705-718.

[37] Parsons, L.M., Temporal and kinematic properties of motor behavior reflected in mentally simulated action, J. Exp. Psychol. Human Perc. Perf., 20 (1994) 709-730.

[38] Passingham, R.E., Premotor cortex and the retrieval of movement, Brain Behav. Evol., 33 (1989) 189-192.

[39] Paus, T., Petrides, M., Evans, A.C. and Meyer, E., Role of the human anterior cingulate cortex in the control of oculomotor, manual and speech responses: a positron emission tomography study, $J$. Neurophysiol., 70 (1993) 453-469.

[40] Pickenhain, L., Towards a holistic conception of movement control.
In H.T.A. Whiting (Ed.), Human Motor Actions: Bernstein Reassessed, North-Holland, Amsterdam, 1984.

[41] Posner, M.I. and Presti, D.E., Selective attention and cognitive control, Trends Neurosci., 10 (1987) 13-17.

[42] Posner, M.I., Petersen, S.E., Fox, P.T. and Raichle, M.E., Localization of cognitive operations in the human brain, Science, 240 (1988) $1627-1631$.

[43] Pylyshyn, Z.W., The imagery debate: analogue media versus tacit knowledge, Psychol. Rev, 88 (1981) 16-45.

[44] Requin, J., Neural basis of movement representations. In J. Requin and G.E. Stelmach (Eds.), Tutorials in motor neurosciences, Nato ASI Series, Kluwer Academic Publisher, Dordrecht, 1991.

[45] Richman, C.L., Mitchell, D.B. and Reznick, J.S., Mental travel: some reservations, J. Exp. Psychol. Human Perc. Perf., 5, 1 (1979) $13-18$.

[46] Roland, P.E., Skinhoj, E., Lassen, N.A. and Larsen, B., Different cortical areas in man in organization of voluntary movements in extrapersonal space, J. Neurophysiol., 43 (1980) 137-150.

[47] Roland, P.E., Metabolic measurements of the working frontal cortex in man, Trends Neurosci., 11 (1984) 430-435.

[48] Ryding, E., Decety, J., Sjöholm, H., Stenberg, G. and Ingvar, D.H., Motor imagery activates the cerebellum regionally. A SPECT rCBF study with ${ }^{99 \mathrm{~m}}$ Tc-HMPAO, Cogn. Brain Res., 2 (1993) 94-99.

[49] Stelmach, G.E. and Hughes, B.G., Cognitivism and future theories of action: basic issues. In W. Prinz and A.F. Sanders (Eds.), Cognition and Motor Processes, Springer, Berlin, 1984.

[50] Stephan, K.M., Fink, G.R., Passingham, R.E., Silbersweig, A.O., Ceballos-Baumann, A.O., Frith, C.D. and Frackowiak, R.S.J., Functional anatomy of the mental representation of upper extremity movements in healthy subjects, J. Neurophysiol., 73 (1993) 373-386.

[51] Tanji, J. and Shima, K., Role for supplementary motor area cells in planning several movements ahead, Nature, 371 (1994) 413-416.

[52] Todor, J.I. and Smiley, A.L., Performance differences between the hands: implications for studying distuption to limb praxis. In E.A. Roy (Ed.), Neuropsychological Studies of Apraxia, Elsevier Science Publishers, Amsterdam, 1985.

[53] Tyszka, J.M., Grafton, S.T., Chew, W., Woods, R.P. and Colletti, P.M., Parceling of mesial frontal motor areas during ideation and movement using functional magnetic resonance imaging at 1.5 Tesla, Ann. Neurol., 35 (1994) 746-749.

[54] Vogt, S., Imagery needs preparation too, Behav. Brain Sci., 17 (1994) 226-227.

[55] Wang, Y. and Morgan, W.P., The effect of imagery perspectives on the psychophysiological responses to imagined exercise, Behat. Brain Res., 52 (1992) 167-174.

[56] Wuyam, B., Moosavi, S.H., Decety, J., Adams, L., Lansing, R.W. and Guz, A., Imagination of dynamic exercise produced ventilatory responses which were more apparent in competitive sportsmen, $J$. Physiol., 482 (1995) 713-724

[57] Yue, G. and Cole, K.J., Strength increases from the motor program: comparison of training with maximal voluntary and imagined muscle contractions, J. Neurophysiol., 67 (1992) 1114-1123. 